\title{
"Management of PPH (Placental Site Bleeding) by a new Haemostatic Suture: In Eleven cases of PPH during Caesarean section".
}

\author{
SADHNA MATHUR ${ }^{1}$ and Megha Solanki² \\ ${ }^{1}$ NIMS Medical College and Hospital \\ ${ }^{2} \mathrm{KD}$ Medical College Hospital and Research Centre
}

November 9, 2020

\begin{abstract}
This study includes 11 cases of PPH seen during Caesarean section by a single surgeon Dr. Sadhna Mathur during the last 7years where this hemostatic suture was used to control PPH from placental site in uterine cavity after the medical measures failed to control the bleeding from placental site(uterine cavity). The bleeding point was localized and a haemostatic suture in the overlying uterine musculature was applied. It was found to be completely effective hence it is being shared through this paper.

"Management of PPH (Placental Site Bleeding) by a new Haemostatic Suture: In Eleven cases of PPH during Caesarean section".

Dr. Sadhna Mathur

Professor \& Head of Department

Department of Obstertics \& Gynaecology

NIMS Medical College \& Hospital

Jaipur (Rajasthan ), India

Email-sadhnamathur19@gmail.com

Phone - 9950996294

Dr. Megha Solanki

Senior Resident, Department of Obstertics \& Gynaecology

K D Medical College \& Hospital

Mathura (U.P), India

Email- honeyevans7@gmail.com

Running Title - Hemostatic suture in PPH during caeserean section

Title: - "Management of PPH (Placental Site Bleeding) by a new Haemostatic Suture: In Eleven cases of PPH during Caesarean section".
\end{abstract}


Concept : - It is an established Anatomical fact that the spiral arterioles pass through the interlacing muscle fibers of uterus. These are the blood supply to placenta for fetal oxygen and nutrition(1) .

After delivery of fetus and placenta effective contraction and retraction of the interlacing muscle fibres of uterus compresses the arterioles passing through it to get a good haemostasis. With this concept in cases of PPH after excluding trauma to genital tract, the bleeding sinus from placental site was searched by Sadhna's four finger technique in the uterine cavity. After locating the bleeder based on the above anatomical concept the overlying area of about one cm squares was taken in a figure of 8 haemostatic suture (Saravi's Suture). This suture invariably resulted in good haemostasis.

Abstract: This study includes 11 cases of PPH seen during Caesarean section by a single surgeon Dr. Sadhna Mathur during the last 7years where this hemostatic suture was used to control PPH from placental site in uterine cavity after the medical measures failed to control the bleeding from placental site(uterine cavity). The bleeding point was localized and a haemostatic suture in the overlying uterine musculature was applied. It was found to be completely effective hence it is being shared through this paper.

Introduction: - Primary post partum hemorrhage is known for its significant role in maternal mortality and morbidity since ages(1) After delivery of fetus and placenta the bleeding from torn placental sinuses is effectively controlled by contraction and retraction of uterine musculature in majority of the cases. But in some cases one or more of theses continue to bleed either due to focal atony or torn sinus not occluded well by this nature's ligature.

\section{Materials and methods}

Study design and sampling: It is a retrospective study from December 2011 to December 2018 conducted in the Department of Obstetrics and Gynecology, NIMS Medical college Jaipur and Kanti Devi Medical College and Research Center Mathura.

\section{Total no. of cases -11}

Inclusion criteria- The study includes cases of major post partum hemorrhage (PPH) following caesarean section, randomly selected, where trickle from uterine cavity persisted despite uterine massage and uterotonics (oxytocin infusion, erogmetrine, carboprost and misoprostol). When bleeding persisted for more than 15-20 minutes despite the conventional medical treatment, the case was considered for exploration of uterine cavity to precisely locate the bleeding point in the cavity, as described below.

Exclusion criteria- Patients with unstable vital signs or diagnosed cases of placenta accreta, with or without invasion, were excluded from the study. Cases of PPH following normal delivery and traumatic $\mathrm{PPH}$ were not included in our study.

All cases were operated by the same surgeon (Prof. Sadhna Mathur), with the assistance of other skilled obstetricians.

Patient particulars and a written consent were obtained from the patient's attendents prior to the intervention.

After delivery of the baby and placenta, routine oxytocin injection in the dose of 5-10 IU intravenous directly and $20 \mathrm{IU}$ in $500 \mathrm{ml}$ Ringer solution over 1 hour was started, and the uterus was exteriorized and checked for bleeding from the uterine cavity if any. The margins of the incision site were also examined for any significant bleeding, and if present, was dealt with separately; either by suture or compression.

In cases of PPH, the uterine cavity was explored for any retained placental pieces and if found were removed,. Exploration for any possible trauma or laceration was done, ruling out traumatic PPH. For cases of atonic PPH, after excluding contraindications, conventional medical treatment was given, which included uterine massage and injection methergin ( $0.2 \mathrm{mg}$ intramuscular, up to 2 doses), injection prostadin (250 mcg intramuscular, up to 3 doses) and misoprostol (1000 mcg per rectal). 
Cases where trickle of blood continued despite giving conservative medical treatment with apparantely contracted uterus for duration of more than 15-20 minutes, were considered for precise localization of the bleeding points, using the technique described below.

\section{Sadhna's Four Finger Technique:}

Technique of localizing bleeding point and hemostasis- To precisely locate the trickling point from the uterine cavity, the cavity was arbitrarily divided into four quadrants and each quadrant was packed with a small sponge separately. The inner surface of lower segment, below the cesarean incision margin, was checked for any bleeding point using a finger tip and a 'figure of 8' hemostatic suture was applied as described below after reflecting the bladder, if necessary.

For localizing bleeding point inside the uterine cavity in upper segment, the sponges were removed sequentially one after the other. As a convention, we followed the sequence of removal of sponges from left lower quadrant first, followed by left upper quadrant. The procedure was repeated for the right side, removing sponge from the right lower quadrant, before removing the sponge from right upper quadrant.

After removal of sponge in each quadrant, it was closely observed for any bleeding from that specific quadrant and bleeding point was localized using the four-finger technique as described. Filling of cavity with blood on removing sponge from one quadrant, indicates that the bleeder lies in that quadrant, which either could be on the anterior wall, or the posterior wall of the uterus. To differentiate between the two, we first cover the inner surface of anterior wall of uterus inside the cavity with the palmer surface of four fingers of our right hand, applying just enough pressure to prevent any bleeding from that surface. The cavity is observed for any collection or trickling of blood, which if present, indicates the source to be from the inner surface of posterior wall of the uterus. The precise location of the bleeding point, if present, is determined by using 'four finger technique' for each surface separately.

In this technique, we cover the suspected bleeding surface with four fingers of our right hand, and lift each finger one by one and observe for any bleeding area in the horizontal plane under each finger, starting from lateral to medial side. On localizing the bleeding point horizontally and securing it beneath one of our fingers, we now proceed to move the finger from above downwards, giving us the exact position of the bleeding point in the vertical plane as well, under that finger.

For achieving haemostasis, we have used synthetic, absorbable Vicryl 1-0 (polyglactin 910) round body, $\frac{1}{2}$ circle $4 \mathrm{~cm}$ needle. Covering the bleeding point, that has been localized, with tip of one of our fingers and guiding the needle, a 'figure of 8' suture (Saravi's Haemostatic Suture) is applied over an approximately $1 \mathrm{~cm}$ square area around the bleeding point, entering through the serosal surface of the uterus and taking near full myometrial thickness, but not entering the uterine cavity. The suture is tied with optimum tension to achieve haemostasis; neither too loose that fails to achieve the haemostasis, nor too tight that cuts through the tissue.

For bleeding sinus localised on posterior wall, uterus was exteriorized and anteverted well for easy placement of suture in the area overlying the bleeding point posteriorly.

\section{Results: -}

In all 11 cases good haemostasis was achieved followed by closure of uterine and abdominal incisions in layers.

All patients were in the age group of 25-35 years

It was $1^{\text {st }}$ caesarean section in 04 cases and

Repeat caesarean section in 07 cases

Number of haemostatic sutures required-

01 Case - 03 Sutures 03 Cases - 02 Sutures 07 Cases - 01 Suture 
Total: 11 Cases -16 Sutures

1. Preoperative haemoglobin was above $09 \mathrm{gm} / \mathrm{dl}$ and only 01 unit of whole blood was transfused post operatively.

2. Only 1case had $\mathrm{Hb}$ of $08 \mathrm{gm} / \mathrm{dl}$ and 2 units of whole blood were transfused.

3. None of the above cases required any other surgical intervention.

4. Post operative period was uneventful in all cases and patients were discharged on $07^{\text {th }}$ day as with other cases.

5. Sites where sutures were placed -

01 suture on fundus towards left cornu leaving the cornual end of fallopian tube.

(b) 04 sutures on anterior surface of lower quadrants of uterine cavity and 04 sutures on the wall of uterus for bleeding from the posterior surface of uterine cavity in lower quadrants. (c) 06 sutures in upper two quadrants of uterine cavity. (d) 01suture was placed on the anterior and inner surface of the lower segment of uterus about $2.5 \mathrm{~cm}$ below and lateral to the left angle of LSCS incision. This particular case was reopened 01 hour after caesearean due to persistant significant trickle of blood in spite of a contracted uterus and 20 IU of syntocinon running in drip for more than 01 hour. Traumatic bleeding was excluded and Saravi's suture was placed as in other cases through the myometrium after localizing the bleeding point with fingers.

9. In all cases a written informed consent was taken from the attendents of patients.

\section{Discussion: -}

In obstetrics practice in cases with atonic PPH when the medical measures fail, the different methods have been devised by different surgeons to stop bleeding.

A study conducted by J. Seror et al. in 2005 (2) found balloon tamponade to be associated with risk of infection and resumption of bleeding after taking it out, in $17.06 \%$ cases in a large series. The patients in whom bleeding restarted were shifted for uterine artery embolization.

Different compression sutures have been devised to oppose the walls of the uterine cavity and also to reduce blood supply to the uterus at the same time, to achieve haemostasis at placental site (3) . Compression sutures practiced are:

1. B Lynch Compression Suture (1)

2. Hayman's Sutures (4)

3. CHO's Square Sutures (5)

4. Mansoura VV sutures (6)

These compression sutures required a skilled obstetrician for the technique as optimum degree of tension to achieve compression of the large bulk of tissue is required while tying the knot without getting cut through.

Saad Benkirane, at all 2017 (7) has reported uterine necrosis following combination of uterine compression sutures with vascular ligation done during postpartum haemorrhage. The other reported complications are pyometra erosion of strap through the uterine wall, uterine ischaemia and synechie. The frequency of complications still remain unclear.

To overcome the aforesaid complications, removable uterine compression sutures were devised by Zhang ZW 2015(8), but they found the method of suture removal to be cumbersome.

The present study includes a limited number of cases but we hope if this method is practiced at a larger level where ever required in future it will be a step overcoming many problems in the management of PPH.

\section{Advantages of this method are:}

1. It is an easy procedure. It only needs learning the technique to locate bleeding point in uterine cavity and to take the suture from serosal surface over it. 
2. The area of uterine musculature taken in the hemostatic suture is about $1 \mathrm{~cm}^{2}$. The area of tissue involved is very small as compared to different compression suture. So the chance of getting cut through, future necrosis, a tight or loose suture is very less.

3. After tying the suture immediate control of bleeding can be seen on the table only

4. Due to early control of bleeding the requirement of blood and blood products is very less.

5. As the uterine cavity is not entered so the risk of synechie formation is not there.

6. As the decision is taken early and sutures are applied there only on the table so the risk of Obstetric shock and its consequences are minimized.

7. The need of procedures like step wise devascularisation, uterine artery embolization and hysterectomy is eliminated.

8. Hospital stay of the patient is equivalent to that of any other caesarean section.

\section{Disadvantages of this method are:}

The only disadvantage is that the technique to localise the bleeding sinus needs to be learned well and this technique is very simple and very easy to learn.

\section{Conclusions: -}

This new method of localizing the bleeding point and applying a haemostatic suture on the overlying uterine muscle was found very effective. The author strongly believes that its practice at a larger level will prove very useful to obstetricians world wide. It will also help us to know any associated problems and complications when practised in large number of cases.

\section{Supporting Information :-}

Two video clips of the technique used are attached.Disclosure if interest :- There is no conflict of interest in authors on any financial, personal, political, intellectual and religious issue.Contribution to authorship :- Author 1 Sadhna Mathur single surgeon while operating cases of cesarean section developed this technique and suture in cases having PPH during cesarean with effective control of bleeding . $2^{\text {nd }}$ Author Megha Solanki has helped to convert this work to this paper with all effortsFunding - No funding from any source was taken what so ever. References: -

1. D.C. Dutta, Text Book of Obstetrics, $9^{\text {th }}$ edition. Hiralal Konar Jaypee health sciences publication: 2018, Chapter-28, Complications of third stage of labour: Pg. 385, 387,392.

2. J. Seror, C. Allauche 83 S. Elhaik. Use of sengstaken - Blakemose tube in massive postpartum haemosshage: a series of 17 cases. Acta Obstet Gynae col of seand 2005; 84: 660-664.

3. Essentials of Obstetrics. Lakshmi Seshadri and Gita Arjun. Fifter Impression 2018. Wolters, Kluwer Health (India) Pvt. Lte., New Delhi Chapter 43. Complications of third stage of Labour. Page No. 641,642,644-646.

4. Hayman RG, Arul Kumaran S, Steer PJ. Uterine Compression sutures : surgical management of postpartum haemorrhage. Obstet Gynecol 2002: 99: 502-506.

5. Cho JH, Jun HS, Lee CN. Hemostatic suturing technique for uterine bleeding during cesarean delivery. Obstet Gynaecol 2000: 96: 129-131.

6. EI Refaesset al. is early intervention using Mansoura - VV uterine compression sutures an effective procedure in the management of primary atonic postpartum haemorrhage? ; a prospective study. BMC pregnancy and childbirth may 2017, 17:160.

7. Saad Benkirane, Hamane Saadi, Badr Serji, Ahmed Mimouni. Uterine necrosis following a combination of uterine compression sutures and vascular ligation during a postpartum haemorrhage, International Journal of Surgery case Reports 38 (2017) 5-7.

8. Zhang $Z W$, Liu $C Y$, Ya $N$, Guo W. Removable uterine compression sutures for postpartum haemorrhage. BJOG 2015: 12: 429-433. 